\title{
Molecule-radiation and molecule-molecule processes in condensed media: a microscopic QED theory
}

\author{
Gediminas Juzeliūnas \\ Institute of Theoretical Physics and Astronomy, A. Goštauto 12, Vilnius, 2600, Lithuania
}

Received 17 January 1995

\begin{abstract}
A microscopic QED theory has been developed for the description of molecule-radiation and molecule-molecule processes in condensed molecular media. Adopting a polariton model, an explicit normal-mode expansion has been derived for the operator of the local displacement field. (It is the local displacement operator that describes the coupling of the individual molecules with the field.) The expansion manifestly embodies refractive influences, including, inter alia, local field contributions. To this end, the local field effects intrinsically emerge within the present formalism that systematically treats Umklapp processes; in addition, the theory accommodates an arbitrary number of molecular levels contributing to the refractive index. The mode expansion is applicable for the investigation of a variety of molecule-radiation and moleculemolecule processes in condensed phases. In the present study, medium-induced modifications are analysed for spontaneous emission and linear absorption; applications to the two-centre (bimolecular) processes are also discussed.
\end{abstract}

\section{Introduction}

Molecular quantum electrodynamics (QED) [1,2] is the most suitable formalism for the description of the interaction of a radiation field with molecules (or atoms) and also of the interaction between the molecules. The QED method has been widely used in studying various phenomena including spontaneous emission $[1,3,4]$, superradiance [3,5], optical activity [1,6-10], harmonic generation [11,12], bimolecular single- or multi-photon processes [13-16], retardation of interactions between the molecules [17-22] and also retardation effects in resonance energy transfer [23-36], etc. A characteristic feature of the QED approach (in its multipolar formulation [1]) is that both the molecule-molecule and also the molecule-radiation processes result exclusively from the coupling of individual molecules with the radiation field. Hence, the coupling between the molecules is fully retarded, mediated by the intermolecular propagation of transverse virtual photons. For instance, the resonance energy transfer [25-36] appears to be a process of the second order of perturbation, involving emission of a virtual (intermediate) photon by one of the participating species and its subsequent recapture by another molecule. In a similar way, other molecule-molecule or molecule-radiation processes are also

\footnotetext{
Dedicated to Professor David P. Craig on the occasion of his 75 th Birthday.
} 
described in terms of creation and annihilation of virtual or real photons by molecules. The number of such events determines the order of a process: under this classification, the ordinary single-photon spontaneous emission is a first-order process [1]; the optical activity for a pair of achiral molecules emerges in the third order $[7,8]$, etc. The perturbational method adequately represents the molecule-radiation and molecule-molecule processes in sufficiently dilute molecular systems. With the increase of the concentration of molecules constituting a surrounding medium, higher-order (indirect) perturbational contributions may become important, in addition to the leading lowest-order diagrams. In this respect, Craig and Thirunamachandran [30] considered effects by a third-body mediation of the resonance energy transfer between a pair of molecules. The microscopic treatment was followed by a discussion of the way to incorporate the dielectric effects. It was suggested from macroscopic arguments [30] that the vacuum dielectric permittivity $\varepsilon_{0}$ entering the rate of excitation transfer in vacuo should be replaced by its medium counterpart $\varepsilon$ to represent the screening.

The present QED study makes use of another alternative approach to the molecule-radiation and moleculemolecule processes in condensed molecular systems. Specifically, the theory is based on a concept under which the molecules interact with the field and also between each other through absorption or emission of photons that are now fully "dressed" by the molecular medium. Such "medium-dressed" photons, known as polaritons [3,37], represent normal modes of mutually coupled material and radiation subsystems, both constituting a single dynamical system (a polariton bath). The theory is fully microscopic, based on an explicit consideration of a discrete molecular medium. A distinctive feature of the present study is that the medium influences, including inter alia screening and local field contributions, now intrinsically emerge in the leading (lowest-order) diagrams, without any consideration of complicated diagrams associated with the indirect processes. In other words, an infinite series of such indirect processes of photon scattering by the molecules of the medium is now fully taken into account in an alternative way through "dressing" of photons, i.e., adopting the polariton concept.

Polaritons are a familiar subject in the solid-state physics [38-43]. In recent years the polariton concept has been addressed to problems of quantisation of radiation in dielectrics [4,44-46]. In this respect, an important aspect is how to represent various operators for the quantised fields in terms of normal polariton modes. Huttner et al. [44-46] considered the normal-mode expansion of macroscopic electromagnetic fields in a uniform dielectric medium, modelled by a harmonic oscillator field. Accordingly, their approach did not reflect the features associated with the discrete molecular medium, such as local field effects. The related study by Ho and Kumar [4] extended the theory to the case of a non-uniform (discrete) medium consisting of quantum oscillators (atoms or molecules) with one resonance frequency. Another development by Knoester and Mukamel [3], also based on the two-level (one resonance frequency) representation of each atom or molecule of the medium, involved a consideration of operators for the microscopic displacement field calculated at the lattice sites, that is, the local field operators. This facilitated the subsequent analysis of polariton-mediated intermolecular processes. Juzeliūnas and Andrews [35,36] studied influences of the surrounding medium on the resonance energy transfer. The transfer process was considered to be mediated by intermolecular propagation of virtual polaritons. In contrast to the related work by Knoester and Mukamel [3], here an arbitrary number of the energy levels (excitation frequencies) has been accommodated for each molecule of the system, including inter alia a situation where the energy levels of the molecules form dense and smooth absorption bands. As a result, the theory $[35,36]$ is applicable to both transparent and absorbing media. The ensuing mathematical complications have been resolved through the development of appropriate Green's function techniques in order to by-pass an explicit consideration of virtual polaritons mediating the process. However, such an approach is not suitable for the present consideration of radiation-molecule and molecule-molecule processes that might involve real incoming and outgoing "dressed" photons in addition to the virtual ones. The present theory will provide an explicit normal-mode expansion of the operator for the local displacement operator in a condensed molecular medium. (It is the local field operator that describes the coupling of individual molecules with the field.) Local field factors and other refractive modifications are to be identified readily in the mode expansion. Again, individual molecules are represented by an arbitrary number of energy levels, so that an arbitrary number of 
dispersion branches arises in the spectrum of normal polariton modes: For comparison, only two dispersion branches are featured in the two-level approaches by Knoester and Mukamel [3] and Ho and Kumar [4].

The paper is organised as follows. In the next section a second-quantised Hamiltonian is defined for a system consisting of a quantised electromagnetic field coupled to a discrete molecular (or atomic) medium. In Section 3.1 a formal diagonalisation is presented, followed by a derivation of normal-mode expansion for the local displacement field operator in Section 3.2. Section 4 illustrates the theory with applications to molecule-radiation and molecule-molecule processes in the condensed phases. These will include spontaneous emission and linear absorption; two-centre (bimolecular) processes are also considered briefly in Section 4. Applications to other processes will appear in a separate study. Finally, the concluding Section 5 summarises the results.

\section{Hamiltonian}

The system of interest comprises an ensemble of molecules (or atoms) and a quantised electromagnetic field. The total Hamiltonian of such a system may be written as

$$
H=H_{\text {rad }}+H_{\text {mol }}+H_{\text {coup }} \text {. }
$$

The first term in this equation is the Hamiltonian for the quantised radiation field. Other terms represent, respectively, the Hamiltonian for the molecular sub-system and the coupling operator: The specific form of the latter operators $H_{\text {mol }}$ and $H_{\text {coup }}$ depends substantially on the QED formulation adopted. In the most familiar minimal coupling formulation of QED $[1,47,48]$, the contributions due to the intermolecular electrostatic interaction feature in the molecular Hamiltonian $H_{\text {mol. }}$. The present study employs another multipolar formulation of QED related to the minimal coupling QED by a canonical transformation $[1,47]$. In such a multipolar formulation, the electrostatic interaction is cancelled between the molecules, intermolecular coupling exclusively being mediated by a quantised radiation field. We also adopt an electric dipole approximation of the multipolar QED: This is normally sufficient for situations where the dimensions of the molecules (or their chromophoric groups) are small compared with both the radiation wavelength and the characteristic intermolecular separations.

Under these conditions, the molecular and coupling Hamiltonians are given by [1]

$$
H_{\mathrm{mol}}=\sum_{\zeta} H_{\zeta},
$$

and

$$
H_{\text {coup }}=\sum_{\zeta} H_{\zeta}^{\text {int }}
$$

with

$$
H_{\zeta}^{\mathrm{int}}=-\varepsilon_{0}^{-1} \boldsymbol{\mu}(\zeta) \cdot \boldsymbol{d}^{\perp}\left(\boldsymbol{r}_{\zeta}\right)
$$

where the summation extends to all the molecules $\zeta$ of the system. The above $H_{\zeta}$ and is the Hamiltonian for a molecule $\zeta$ positioned at $\boldsymbol{r}_{\zeta}, H_{\zeta}^{\text {int }}$ is the Hamiltonian for the molecule-field interaction, and $\boldsymbol{\mu}(\zeta)$ is the operator for the electric dipole moment of $\zeta$. Finally, $\boldsymbol{d}^{\perp}\left(\boldsymbol{r}_{\zeta}\right)$ is the operator for the electric displacement field calculated at the molecule site $\boldsymbol{r}_{\zeta}$, i.e., $\boldsymbol{d}^{\perp}\left(\boldsymbol{r}_{\zeta}\right)$ represents the operator for the local displacement field. The displacement operator may be cast in terms of photon creation and annihilation operators, $\boldsymbol{a}^{\dagger(\lambda)}(\boldsymbol{k}+\boldsymbol{G})$ and $a^{(\lambda)}(\boldsymbol{k}+\boldsymbol{G})$, as

$$
\boldsymbol{d}^{\perp}\left(\boldsymbol{r}_{\zeta}\right)=\mathrm{i} \sum_{\boldsymbol{k}, \boldsymbol{G}} \sum_{\lambda=1}^{2}\left(\frac{\hbar c|\boldsymbol{k}+\boldsymbol{G}| \varepsilon_{0}}{2 V_{0}}\right)^{1 / 2} \boldsymbol{e}^{(\lambda)}(\boldsymbol{k}+\boldsymbol{G})\left[a^{(\lambda)}(\boldsymbol{k}+\boldsymbol{G})-a^{\dagger(\lambda)}(-\boldsymbol{k}-\boldsymbol{G})\right] \mathrm{e}^{\mathrm{i} \boldsymbol{k} \cdot \boldsymbol{r}_{\zeta}}
$$

where for convenience the photon wave-vector $k+G$ has been partitioned into the first Brillouin zone vector $k$ and the inverse lattice vector $G$ to reflect the lattice symmetry imposed at this stage on the molecular 
sub-system. (Since the molecules are supposed to occupy the lattice sites, an exponential factor $\mathrm{e}^{\mathrm{i} G \cdot r_{\zeta}}(=1)$ is missing in the above expansion.) Here also $V_{0}$ is the quantisation volume and $\boldsymbol{e}^{(\lambda)}(\boldsymbol{k}+\boldsymbol{G})$ is the unit vector for photon polarisation $(\lambda=1,2)$ : the latter is chosen to be real and such that $e^{(\lambda)}(-\boldsymbol{k}-\boldsymbol{G})=\boldsymbol{e}^{(\lambda)}(\boldsymbol{k}+\boldsymbol{G})$. In a similar way, the expanded radiation Hamiltonian reads

$$
H_{\mathrm{rad}}=\sum_{\boldsymbol{k}, \boldsymbol{G}} \sum_{\lambda=1}^{2} \hbar c|\boldsymbol{k}+\boldsymbol{G}|\left[\boldsymbol{a}^{\dagger(\lambda)}(\boldsymbol{k}+\boldsymbol{G}) a^{(\lambda)}(\boldsymbol{k}+\boldsymbol{G})+a^{(\lambda)}(\boldsymbol{k}+\boldsymbol{G}) a^{\dagger(\lambda)}(\boldsymbol{k}+\boldsymbol{G})\right] .
$$

To complete the definition of the system, the Hamiltonian for the molecule $\zeta$ and the molecular dipole operator may be expressed in second quantisation, as

$$
\begin{aligned}
& H_{\zeta}=\sum_{\gamma} \sum_{j=1}^{3} \hbar \Omega_{\gamma} B_{\zeta, \gamma, j}^{\dagger} B_{\zeta, \gamma, j}+E_{\mathrm{g}}^{\zeta}, \\
& \boldsymbol{\mu}\left(\boldsymbol{r}_{\zeta}\right)=\sum_{\gamma} \sum_{j=1}^{3}\left(B_{\zeta, \gamma, j}^{\dagger}+B_{\zeta, \gamma, j}\right) \mu_{\gamma} \boldsymbol{e}_{j},
\end{aligned}
$$

where $E_{\mathrm{g}}^{\zeta}$ denotes the ground-state energy for the molecular sub-system, $\boldsymbol{e}_{j}$ is the unit vector along a Cartesian axis $j$, and $B_{\zeta, \gamma, j}^{\dagger}\left(B_{\zeta, \gamma, j}\right)$ is the Bose operator for creation (annihilation) of excitation at a molecule $\zeta^{1}$. When acting on a ground molecular state, the creation operator $B_{\zeta, \gamma, \lambda^{\prime}}^{\dagger}$, promotes the molecule $\zeta$ to an excited electronic state, characterised by indexes $\gamma$ and $j$. An arbitrary number of excitation frequencies $\Omega_{\gamma}$, has been accommodated for each individual molecule. An additional to $\gamma$ index $j=1,2,3$ explicitly refers to the triple degeneracy of excited molecular states, the associated transition dipole moments $\mu_{\gamma} e_{j}$ being mutually perpendicular. (For convenience, the transition dipoles $\mu_{\gamma}$ are chosen to be real.)

In this way, the molecules have been assumed to be characterised by isotropic (or nearly isotropic) polarisabilities. Such a model may also describe a common situation where non-isotropic species are randomly oriented in their sites. As regards the spatial arrangement, the molecules are supposed to constitute a simple cubic lattice. Yet, the main results to come seem to be not sensitive to the possible lack of translational symmetry: For the potential applications of the theory to various molecule-radiation processes, such as photon absorption and emission, the transition frequencies of the embedded species are normally situated far away from the exciton resonances of the material medium so that the effects of translation symmetry are of no significant importance.

Transforming the molecular operators to the momentum space,

$$
\begin{aligned}
& B_{k, \gamma, j}=N^{-1 / 2} \sum_{\zeta} B_{\zeta, \gamma, j} \mathrm{e}^{-\mathrm{i} \boldsymbol{k} \cdot \boldsymbol{r}_{\zeta},} \\
& B_{k, \gamma, \lambda^{\prime}}=\sum_{j=1}^{3}\left[\boldsymbol{e}^{\left(\lambda^{\prime}\right)}(\boldsymbol{k}) \cdot \boldsymbol{e}_{j}\right] B_{k, \gamma, j},
\end{aligned}
$$

$\left(\lambda^{\prime}=1,2,3\right.$ with $e^{\left(\lambda^{\prime}\right)}(\boldsymbol{k})=\hat{\boldsymbol{k}} \equiv \boldsymbol{k} / \boldsymbol{k}$, for $\lambda^{\prime}=3$ ), the molecular Hamiltonian (2.2) and the coupling operator (2.3) become, through Eqs. (2.4), (2.5) and (2.7)

$$
H_{\mathrm{mol}}=\sum_{k, \gamma} \sum_{\Lambda^{\prime}=1}^{3} \hbar \Omega_{\gamma}\left(B_{k, \gamma, \lambda^{\prime}}^{\dagger} B_{k, \gamma, \lambda^{\prime}}+B_{k, \gamma, \lambda^{\prime}} B_{k, \gamma, \Lambda^{\prime}}^{\dagger}\right)
$$

\footnotetext{
${ }^{1}$ At this stage, the Bose approximation is adopted for the molecular creation and annihilation operators, replacing the exact Pauli commutation rules by those of the Bose type. This is nonetheless a common approach (see, e.g., Refs. [3,35,37]), producing a good model representation for three-dimensional molecular structures at relatively low fraction of excited molecules in the system.
} 
and

$$
\begin{aligned}
H_{\text {coup }}= & \sum_{\boldsymbol{k}, \boldsymbol{G}, \gamma} \sum_{\lambda^{\prime}=1}^{3} \sum_{\lambda=1}^{2} \hbar\left[C_{\left(\boldsymbol{k}, \gamma, \lambda^{\prime}\right)(k, \boldsymbol{G}, \lambda)}\left(B_{\boldsymbol{k}, \gamma, \lambda^{\prime}}^{\dagger}+B_{-\boldsymbol{k}, \gamma, \lambda^{\prime}}\right) a^{(\lambda)}(\boldsymbol{k}+\boldsymbol{G})\right. \\
& \left.+C_{\left(\boldsymbol{k}, \gamma, \lambda^{\prime}\right)(\boldsymbol{k}, \boldsymbol{G}, \lambda)}^{*} a^{\dagger(\lambda)}(\boldsymbol{k}+\boldsymbol{G})\left(B_{k, \gamma, \lambda^{\prime}}+B_{-\boldsymbol{k}, \gamma, \lambda^{\prime}}^{\dagger}\right)\right]
\end{aligned}
$$

where

$$
C_{\left(k, \gamma, \lambda^{\prime}\right)(k, G, \lambda)}=-\mathrm{i}\left(\frac{c|k+G|}{2 \varepsilon_{0} \hbar} \rho\right)^{1 / 2} \mu_{\gamma}\left[\boldsymbol{e}^{\left(\lambda^{\prime}\right)}(\boldsymbol{k}) \cdot \boldsymbol{e}^{(\lambda)}(\boldsymbol{k}+\boldsymbol{G})\right]
$$

is the radiation-matter coupling matrix,

$$
\rho=N / V_{0}
$$

being the mean number density and $N$ being the number of molecules in a quantisation volume $V_{0}$. The coupling Hamiltonian (2.12) manifestly accommodates photon Umklapp processes $(\boldsymbol{k} \rightarrow \boldsymbol{k}+\boldsymbol{G})$. A specific zero-point energy $E_{\mathrm{g}}$ has been chosen in the molecular Hamiltonian (2.11) to make it symmetric with respect to the molecular creation and annihilation operators. Eqs. (2.11) and (2.12), together with (2.1) and (2.6) define the full second-quantised Hamiltonian $H$. Strictly speaking, the Hamiltonian $H$ should also contain a field-independent contribution, represented by the last term of Eq. (3.6.31) of Ref. [1]. Yet, such a contribution, essential for the Lamb shift calculations, is not important for the present purposes.

\section{Normal-mode representation of local displacement field}

Eq. (2.5) casts the operator $\boldsymbol{d}^{\perp}\left(\boldsymbol{r}_{\zeta}\right)$ for the local displacement field in terms of operators for the creation and annihilation of photons. It is to be pointed out that these photons are already not entirely "pure" vacuum photons, since the familiar canonical transformation [1], relating the original minimal coupling to the present multipolar QED formulations, entails some mixing between the quantised radiation field and the matter. Yet, the photons also do not represent the normal modes of radiation field in a medium. The full system contains a sub-system of densely packed molecules, in addition to a quantised radiation field, both sub-systems experiencing substantial interaction due to the coupling Hamiltonian (2.3) or (2.12). Consequently, the radiation field and the molecular medium constitute a single dynamical system. The normal modes of such a combined system are represented by new quasi-particles known as polaritons [3,35,37].

\subsection{Formal diagonalisation}

Adopting a new set of Bose operators for polariton creation $\left(P_{\sigma}^{\dagger}\right)$ and annihilation $\left(P_{\sigma}\right)$, the total Hamiltonian acquires a diagonal form

$$
H=\frac{\hbar}{2} \sum_{\sigma} \Pi_{\sigma}\left(P_{\sigma}^{\dagger} P_{\sigma}+P_{\sigma} P_{\sigma}^{\dagger}\right)
$$

where $\Pi_{\sigma}$ is an eigen-frequency of polariton, index $\sigma$ labelling the normal polariton modes ${ }^{2}$. The above polariton operators are related to an initial set $A_{\alpha}$ of molecular and radiative operators,

$$
\begin{aligned}
A_{\alpha} & =a^{(\lambda)}(\boldsymbol{k}+\boldsymbol{G}), & & \text { for } \alpha=(\boldsymbol{k}, \boldsymbol{G}, \lambda), \\
& =B_{k, \gamma, \lambda^{\prime}}, & & \text { for } \alpha=\left(\boldsymbol{k}, \gamma, \lambda^{\prime}\right),
\end{aligned}
$$

\footnotetext{
${ }^{2}$ In this section, the quasi-momentum $k$ will be implicitly accommodated within the polariton index $\sigma$.
} 
through the following transformation of the Bogliubov-Tyablikov type:

$$
\begin{aligned}
& P_{\sigma}=\sum_{\alpha}\left[(\sigma, 1|S| \alpha, 1) A_{\alpha}+(\sigma, 1|S| \alpha, 2) A_{\tilde{\alpha}}^{\dagger}\right], \\
& P_{\tilde{\sigma}}^{\dagger}=\sum_{\alpha}\left[(\sigma, 2|S| \alpha, 1) A_{\alpha}+(\sigma, 2|S| \alpha, 2) A_{\tilde{\alpha}}^{\dagger}\right],
\end{aligned}
$$

where $S$ is a transformation matrix, $\mid \alpha, 1)$ and $\mid \alpha, 2),((\sigma, 1 \mid$ and $(\sigma, 2 \mid)$ being the unit row (column) matrix-vectors; for more details on the matrix formulation of the polariton problem see Ref. [35]. Here also the tildes indicate momentum reversals:

$$
\left(\widetilde{k, \gamma, \lambda^{\prime}}\right)=\left(-\boldsymbol{k}, \gamma, \lambda^{\prime}\right), \quad(\widetilde{\boldsymbol{k}, \boldsymbol{G}, \lambda})=(-\boldsymbol{k},-\boldsymbol{G}, \lambda)
$$

Performing an inverse transformation back to the radiative operators,

$$
\begin{aligned}
& a^{(\lambda)}(\boldsymbol{k}+\boldsymbol{G})=\sum_{\sigma}\left[\left(\boldsymbol{k}, \boldsymbol{G}, \lambda, 1\left|S^{-1}\right| \sigma, 1\right) P_{\sigma}+\left(\boldsymbol{k}, \boldsymbol{G}, \lambda, 1\left|S^{-1}\right| \sigma, 2\right) P_{\tilde{\sigma}}^{\dagger}\right], \\
& a^{\dagger(\lambda)}(-\boldsymbol{k}-\boldsymbol{G})=\sum_{\sigma}\left[\left(\boldsymbol{k}, \boldsymbol{G}, \lambda, 2\left|S^{-1}\right| \sigma, 1\right) P_{\sigma}+\left(\boldsymbol{k}, \boldsymbol{G}, \lambda, 2\left|S^{-1}\right| \sigma, 2\right) P_{\tilde{\sigma}}^{\dagger}\right],
\end{aligned}
$$

the linear combination of photon creation and annihilation operators, featured in the mode expansion (2.5), may be expressed through the polariton Bose operators, as

$$
\begin{aligned}
a^{(\lambda)}(\boldsymbol{k}+\boldsymbol{G})-\boldsymbol{a}^{\dagger(\lambda)}(-\boldsymbol{k}-\boldsymbol{G})= & \sum_{\sigma}\left\{\left[\left(\boldsymbol{k}, \boldsymbol{G}, \lambda, \mathbf{1}\left|-(\boldsymbol{k}, \boldsymbol{G}, \lambda, 2 \mid] S^{-1}\right| \sigma, 1\right) P_{\sigma}\right.\right. \\
& \left.-(\sigma, 1|Q S Q[\mid-\boldsymbol{k},-\boldsymbol{G}, \lambda, 1)-|-\boldsymbol{k},-\boldsymbol{G}, \lambda, 2)] P_{\sigma}^{\dagger}\right\},
\end{aligned}
$$

where

$$
S^{-1}=Q^{\prime} S^{*} Q
$$

is the matrix for the inverse transformation [35,37], with

$$
Q=\left(\begin{array}{cc}
1 & 0 \\
0 & -1
\end{array}\right)
$$

being a diagonal matrix, i.e., $\left.Q \mid \sigma, j)=(-1)^{j+1} \mid \sigma, j\right)(j=1,2)$. Furthermore, in writing Eq. (3.8), the use has been made of the following conditions imposed on the transformation matrix $S$ [35]:

$$
\begin{aligned}
& (\tilde{\sigma}, i|S| \tilde{\alpha}, j)=(\sigma, i|S| \alpha, j), \quad(i, j=1,2), \\
& \left(\sigma, 1\left|S^{*}\right| \alpha, 1\right)=(\sigma, 2|S| \alpha, 2), \\
& \left(\sigma, 1\left|S^{*}\right| \alpha, 2\right)=(\sigma, 2|S| \alpha, 1) .
\end{aligned}
$$

The operator for the local displacement field may now be formally represented through the polariton Bose operators. Substituting Eq. (3.8) into (2.5), one finds for the Cartesian components of the local displacement field:

$$
d_{j}^{\perp}\left(\boldsymbol{r}_{\zeta}\right)=\mathrm{i} \hbar \varepsilon_{0} N^{-1 / 2} \sum_{\sigma}\left[{ }_{j}\left(k, \operatorname{rad}\left|S^{-1}\right| \sigma, 1\right) P_{\sigma} \mathrm{e}^{\mathrm{i} \boldsymbol{k} \cdot \boldsymbol{r}_{\zeta}}-(\sigma, 1|Q S Q| k, \mathrm{rad})_{j} P_{\sigma}^{\dagger} \mathrm{e}^{-\mathrm{i} \boldsymbol{k} \cdot \boldsymbol{r}_{\zeta}}\right],
$$

$(j=1,2,3)$, where

$$
\left.\left.\mid \boldsymbol{k}, \mathrm{rad})_{j}=\sum_{\boldsymbol{G}} \sum_{\lambda=1}^{2}[\mid \boldsymbol{k}, \boldsymbol{G}, \lambda, 1)-\mid \boldsymbol{k}, \boldsymbol{G}, \lambda, 2\right)\right] e_{j}^{(\lambda)}(\boldsymbol{k}+\boldsymbol{G})\left(\frac{c|\boldsymbol{k}+\boldsymbol{G}|}{2 \varepsilon_{0} \hbar} \rho\right)^{1 / 2}
$$

is the radiative raw matrix, $(k, \mathrm{rad} /$ being its column counterpart. Here the summation over the inverse lattice vector $\boldsymbol{G}$ represents the photon Umklapp processes: These processes are essential for a proper description of local fields within the framework of the present multipolar formulation of QED. 


\subsection{Explicit results}

To deal with the matrix elements of $S$ entering the mode expansions (3.14) consider the following Green function:

$$
\begin{aligned}
\ddot{G}_{l j}^{\mathrm{rad}}(k, \omega)= & \sum_{\boldsymbol{k}, \boldsymbol{m}, \lambda}\left(\frac{{ }_{l}\left(\boldsymbol{k}, \mathrm{rad}\left|S^{-1}\right| \boldsymbol{k}, \boldsymbol{m}, \lambda, 1\right)(\boldsymbol{k}, m, \lambda, 1|Q S Q| \boldsymbol{k}, \mathrm{rad})_{j}}{\omega-\omega_{k}^{(m)}+\mathrm{i} \eta}\right. \\
& \left.+\frac{{ }_{l}\left(\boldsymbol{k}, \mathrm{rad}\left|S^{-1}\right| \boldsymbol{k}, \boldsymbol{m}, \lambda, 2\right)(\boldsymbol{k}, m, \lambda, 2|Q S Q| \boldsymbol{k}, \mathrm{rad})_{j}}{-\omega-\omega_{k}^{(m)}-\mathrm{i} \eta}\right), \quad(\eta \rightarrow+0),
\end{aligned}
$$

with

$$
\omega_{k}^{(m)} \equiv \Pi_{\sigma}, \quad m=1,2,3, \ldots
$$

denoting the polariton eigen-frequencies. At this stage, the $\boldsymbol{k}$ dependence has been explicitly displayed in the mode index $\sigma \equiv(k, m, \lambda)$; another sub-index $m$ will refer to a branch $(m=1,2,3, \ldots)$ of polariton dispersion, the third sub-index $\lambda$ being reserved to label the degenerate sub-levels within the branch. For each eigen-frequency $\omega_{k}^{(m)}$, one finds

$$
\sum_{\lambda}\left(\boldsymbol{k}, \operatorname{rad}\left|S^{-1}\right| \boldsymbol{k}, m, \lambda, 1\right)(\boldsymbol{k}, m, \lambda, 1|Q S Q| \boldsymbol{k}, \operatorname{rad})_{j}=\lim _{\omega \rightarrow \omega_{k}^{(m)}}\left(\omega-\omega_{k}^{(m)}\right) \ddot{G}_{l j}^{\mathrm{rad}}(\omega) .
$$

Eq. (3.18) relates the matrix elements $\left(k, \mathrm{rad}\left|\mathrm{S}^{-1}\right| \boldsymbol{k}, m, \lambda, 1\right)$ of interest and their complex conjugate counterparts

$$
(\boldsymbol{k}, \boldsymbol{m}, \lambda, 1|Q S Q| \boldsymbol{k}, \mathrm{rad})_{j}=\left[j\left(\boldsymbol{k}, \mathrm{rad}\left|S^{-1}\right| \boldsymbol{k}, \boldsymbol{m}, \lambda, \mathbf{1}\right)\right]^{*}
$$

to the Green function $\ddot{G}_{l j}^{\text {rad }}(\omega)$. For positive frequencies $(\omega>0)$, the latter Green function has been determined previously in Eq. (B8) of Ref. [35] to yield

$$
\ddot{G}_{l j}^{\mathrm{rad}}(\boldsymbol{k}, \omega)=\frac{\rho}{3 \hbar \varepsilon_{0}} \frac{1}{n^{2}}\left(\frac{n^{2}+2}{3}\right)^{2}\left(\frac{\left[2 n^{2}(\omega / c+\mathrm{i} \eta)^{2}+k^{2}\right] \delta_{l j}-3 k_{l} k_{j}}{n^{2}(\omega / c+\mathrm{i} \eta)^{2}-k^{2}}-\frac{2 \alpha \rho}{3 \varepsilon_{0}} \delta_{l j}\right), \quad(\eta \rightarrow+0)
$$

subject to the below condition (3.23), with

$$
n^{2} \equiv \varepsilon_{\mathrm{r}}=1+\frac{\alpha \rho / \varepsilon_{0}}{1-\alpha \rho / 3 \varepsilon_{0}}
$$

and

$$
\alpha \equiv \alpha(\omega)=\frac{1}{\hbar} \sum_{\gamma}\left(\frac{\mu_{\gamma}^{2}}{\Omega_{\gamma}-\omega-\mathrm{i} \eta}+\frac{\mu_{\gamma}^{2}}{\Omega_{\gamma}+\omega+\mathrm{i} \eta}\right) .
$$

The above $n \equiv n(\omega)$ is the refractive index of the medium, $\alpha(\omega)$ is the molecular polarisability, and $\varepsilon_{\mathrm{r}} \equiv \varepsilon_{\mathrm{r}}(\omega)=\varepsilon / \varepsilon_{0}$ is the relative dielectric permittivity that apparently satisfies the Clausius-Mossotti relation due to the systematic treatment of photon Umklapp processes. Note that, the effects of the spatial dispersion (dependence of $\varepsilon_{\mathrm{r}}$ on the wavevector $k$ ) are out of the scope of the present study. Finally, Eq. (3.20) holds for [35]

$$
k \ll 2 \pi\left(N / V_{0}\right)^{1 / 3} \equiv 2 \pi / a .
$$

This condition is fully appropriate for the description of the infrared, optical or ultraviolet modes of light in condensed media: In these regions of spectrum, the wavelength $\lambda=2 \pi / k$ exceeds greatly the characteristic 


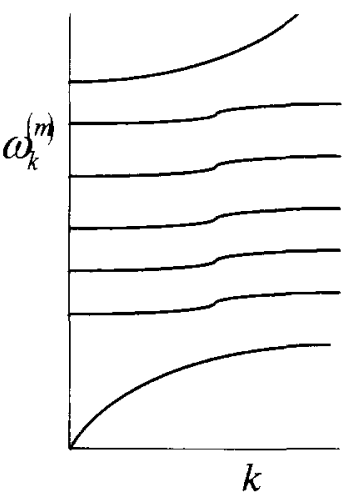

Fig. 1. Schematic representation of polariton dispersion. The dispersion curves embody both the exciton-like and the photon-like branches. As discussed in the text, our subsequent analysis will concentrate mostly on the latter photonic branches.

distance $a$ between the neighbouring species of a condensed molecular system, so that the description in terms of the dielectric constant is appropriate.

In what follows, our analysis will concentrate on the transverse normal modes of the system. Only these modes contribute to the transverse operator for the displacement field. The corresponding poles of the Green function $\ddot{G}_{l j}^{\mathrm{rad}}(\boldsymbol{k}, \omega)$ result from the contribution by the first term in the figure brackets of the Eq. (3.20), giving

$$
\omega_{k}^{(m)}=c k / n\left(\omega_{k}^{(m)}\right) \text {. }
$$

As depicted in Fig. 1, several normal frequencies $\omega_{k}^{(m)} \equiv \omega_{k}^{(m)}(m=1,2, \ldots, M)$ are associated with each value of $k$ : The total number of such dispersion branches is $M=M_{\mathrm{mol}}+1, M_{\text {mol }}$ being the number of molecular frequencies $\Omega_{\gamma}$ involved ${ }^{3}$. For instance, the single-frequency (Hopfield) model considered by Knoester and Mukamel [3] and also Ho and Kumar [4], gives two polariton branches. As a number of molecular frequencies increases, the branches may start to form dense sets of dispersion curves. Hence, the present approach may accommodate the quasi-continuums in the molecular absorption spectra as well. In this way, the current model may adequately represent a wide variety of media, including both lossless and absorbing ones.

Calling on Eqs. (3.18), (3.20) and (3.24), one arrives at

$$
\begin{gathered}
\sum_{\lambda}\left(k, \operatorname{rad}\left|S^{-1}\right| \boldsymbol{k}, m, \lambda, 1\right)(\boldsymbol{k}, m, \lambda, 1|Q S Q| \boldsymbol{k}, \operatorname{rad})_{j} \\
=\frac{\rho \omega_{k}^{(m)} v_{\mathrm{g}}\left(\omega_{k}^{(m)}\right)}{2 \varepsilon_{0} \hbar c n\left(\omega_{k}^{(m)}\right)}\left(\frac{\left[n\left(\omega_{k}^{(m)}\right)\right]^{2}+2}{3}\right)^{2}\left(\delta_{l j}-\hat{k}_{l} \hat{k}_{j}\right),
\end{gathered}
$$

where

$$
v_{\mathrm{g}}(\omega)=c\left(\frac{\mathrm{d}[\omega n(\omega)]}{\mathrm{d} \omega}\right)^{-1},
$$

may be identified to be the group velocity of polariton. Note that both the group velocity and the refractive index are to be calculated at the polariton frequency $\omega_{k}^{(m)}$ in Eq. (3.25).

\footnotetext{
${ }^{3}$ The present study does not consider the polariton branches with extremely high frequencies: These branches, ranging in the areas of photon Umklapp frequencies $c G$ (with $G \neq 0$ ), are well above the frequencies of infrared, optical or ultraviolet modes of interest.
} 
Solutions of Eq. (3.25) are characterised by two polarisation components $\lambda=1,2$ for each frequency $\omega_{k}^{(m)}$ :

$$
\begin{aligned}
{ }_{j}\left(\boldsymbol{k}, \operatorname{rad}\left|S^{-1}\right| \boldsymbol{k}, \boldsymbol{m}, \lambda, 1\right) & =\left[(\boldsymbol{k}, \boldsymbol{m}, \lambda, 1|Q S Q| \boldsymbol{k}, \mathrm{rad})_{j}\right]^{*} \\
& =\left(\frac{\rho \omega_{k}^{(m)} v_{\mathrm{g}}\left(\omega_{k}^{(m)}\right)}{2 \varepsilon_{0} \hbar c n\left(\omega_{k}^{(m)}\right)}\right)^{1 / 2}\left(\frac{\left[n\left(\omega_{k}^{(m)}\right)\right]^{2}+2}{3}\right) e_{j}^{(\lambda)}(\boldsymbol{k}),
\end{aligned}
$$

where we have used the result

$$
\sum_{\lambda=1}^{2} e_{l}^{(\lambda)}(k) \cdot e_{j}^{(\lambda)}(k)=\left(\delta_{l j}-\hat{k}_{l} \hat{k}_{j}\right)
$$

with

$$
e^{(\lambda)}(k) \perp k, \quad \lambda=1,2,
$$

being the unit polarisation vector, already featured in Eq. (2.5). The explicit mode representation of the local displacement operator is now straightforward. Substituting Eq. (3.27) into (3.14), $\boldsymbol{d}^{\perp}\left(\boldsymbol{r}_{\zeta}\right)$ takes the form

$$
\begin{aligned}
& \boldsymbol{d}^{\perp}\left(\boldsymbol{r}_{\zeta}\right) \\
& \quad=\mathrm{i} \sum_{\boldsymbol{k}} \sum_{m} \sum_{\lambda=1}^{2}\left(\frac{\varepsilon_{0} \hbar \omega_{k}^{(m)} v_{\mathrm{g}}\left(\omega_{k}^{(m)}\right)}{2 c V_{0} n\left(\omega_{k}^{(m)}\right)}\right)^{1 / 2}\left(\frac{\left[n\left(\omega_{k}^{(m)}\right)\right]^{2}+2}{3}\right) \boldsymbol{e}^{(\lambda)}(\boldsymbol{k})\left(\mathrm{e}^{\mathrm{i} \boldsymbol{k} \cdot \boldsymbol{r}_{\zeta}} P_{\boldsymbol{k}, m, \lambda}-\mathrm{e}^{-\mathrm{i} \boldsymbol{k} \cdot \boldsymbol{r}_{\zeta}} P_{k, m, \lambda}^{+}\right) .
\end{aligned}
$$

The above summation over $k$ extends to $k \ll 2 \pi / a$ : As discussed earlier in the paper, this restriction is fully appropriate for the consideration of infrared, optical and ultraviolet modes of radiation in condensed molecular systems. The mode expansion (3.30) also involves summations over the branches of polariton dispersion. The emerging in the expansion refractive index and group velocity depends on the branch index $m$ through their argument $\omega=\omega_{k}^{(m)}$. Note that the local field contributions $\left(n^{2}+2\right) / 3$ are explicitly displayed in the mode expansion.

\section{Applications to molecule-radiation and molecule-molecular processes in media}

\subsection{Single-centre processes}

To illustrate the theory, consider first quantum processes that involve a guest molecule A substituting at one of the lattice sites of the host molecular medium. The corresponding Hamiltonian for the molecule-field interaction is given by

$$
V=-\varepsilon_{0}^{-1} \boldsymbol{\mu}(\mathrm{A}) \cdot \boldsymbol{d}^{\perp}\left(\boldsymbol{r}_{\mathrm{A}}\right)
$$

where $\boldsymbol{\mu}(\mathrm{A})$ is the dipole moment operator of the embedded molecule. By making use of the normal-mode expansion (3.30) for the local displacement operator $d^{\perp}\left(\boldsymbol{r}_{\zeta}\right)$ (with $\boldsymbol{r}_{\zeta}=\boldsymbol{r}_{\mathrm{A}}$ ), one can analyse various emission, absorption or scattering processes by molecules embedded in a dielectric medium. Below we will present the simplest examples including the spontaneous emission and linear absorption.

\subsubsection{Spontaneous emission}

Consider a quantum transition by a molecule $A$ from its excited state $\left|A^{*}\right\rangle$ to some lower-lying state $|A\rangle$. The molecule is surrounded by a polariton bath, i.e., the quantised radiation field coupled to the molecules of the medium. The bath is initially in its ground (vacuum) state $|0\rangle$. Subsequently, the bath is promoted to the 


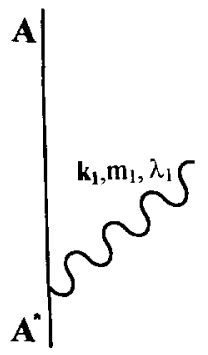

Fig. 2. Spontaneous emission.

final state $P_{k, m, \lambda}^{+}|0\rangle$ due to spontaneous emission of a medium-dressed photon (a bath polariton) by the molecule A, as shown in Fig. 2. The initial and final state-vectors of the process are then

$$
|I\rangle=\left|\mathrm{A}^{*}\right\rangle|0\rangle, \quad|F\rangle=|\mathrm{A}\rangle P_{k, m, \lambda}^{+}|0\rangle .
$$

Note that the above quantum-mechanical bra and ket state-vectors should not be confused with the auxiliary matrix-vectors, such as $\mid \sigma, i)_{\text {and }} \mid \boldsymbol{k}$, rad), featured in the previous sections. Applying the Fermi Golden Rule, the rate of such a spontaneous polariton emission reads

$$
\begin{aligned}
W\left(\mathrm{~A} \leftarrow \mathrm{A}^{*}\right) & =\frac{2 \pi}{\hbar} \sum_{k, m} \sum_{\lambda=1}^{2} \varepsilon_{0}^{-2}\left|\boldsymbol{\mu}_{\mathrm{A}^{*}} \cdot\left\langle 0\left|P_{k, m, \lambda} d\left(r_{\mathrm{A}}\right)\right| 0\right\rangle\right|^{2} \delta\left(\hbar \omega_{\mathrm{A}^{*}}-\hbar \omega_{k}^{(m)}\right) \\
& =\frac{2 \pi \mu_{\mathrm{A}^{*}}^{2}}{3 \varepsilon_{0} c V_{0}} \sum_{k, m} \frac{\omega_{k}^{(m)} v_{\mathrm{g}}\left(\omega_{k}^{(m)}\right)}{n\left(\omega_{k}^{(m)}\right)}\left(\frac{\left[n\left(\omega_{k}^{(m)}\right)\right]^{2}+2}{3}\right)^{2} \delta\left(\hbar \omega_{\mathrm{A}^{*}}-\hbar \omega_{k}^{(m)}\right),
\end{aligned}
$$

where

$$
\boldsymbol{\mu}_{\mathrm{A}^{*}}=\left\langle\mathrm{A}|\boldsymbol{\mu}(\mathrm{A})| \mathrm{A}^{*}\right\rangle
$$

is an abbreviation for the transition dipole moment of $\mathrm{A}, \mu_{\mathrm{A}^{*}}$ being its absolute value. Here also $\omega_{\mathrm{A}^{*}}=\left(\varepsilon_{\mathrm{A}^{*}}-\right.$ $\left.\varepsilon_{\mathrm{A}}\right) / \hbar$ is the resonance frequency of molecule $\mathrm{A}$, and the summation is over the final polariton states characterised by the quasi-momentum $k$, the polarisation $\lambda$ and the branch index $m$.

We shall assume that the emission frequency $\omega_{\mathrm{A}}$ : is situated in a transparent region of the dielectric, away from any exciton-like branches of polariton dispersion ${ }^{4}$. In other words, the molecular frequency $\omega_{\mathrm{A}^{*}}$. is considered to be in resonance with the frequencies $\omega_{k}^{(m)}$ belonging to a photon-like branch $m$. Performing in Eq. (4.4) summation over wave-vectors of emitted polaritons (medium-dressed photons), one arrives to

$$
W\left(\mathrm{~A} \leftarrow \mathrm{A}^{*}\right)=n\left(\frac{n^{2}+2}{3}\right)^{2} \frac{\omega_{\mathrm{A}^{*}}^{3} \mu_{\mathrm{A}^{*}}^{2}}{3 \pi \varepsilon_{0} \hbar c},
$$

with $n \equiv n\left(\omega_{\mathrm{A}^{*}}\right)$, where the group velocity $v_{\mathrm{g}}\left(\omega_{k}^{(m)}\right)$ cancels out during the summation over the polariton wave-vector $k$. In passing we note that the total emission rate may now be obtained readily by averaging over the initial and summing over the final molecular sub-levels (vibrational, rotational, etc.), labelled by indexes $s$ and $q$, as

$$
W^{\mathrm{em}}=\sum_{s, q} \rho_{s} W\left(\mathrm{~A}_{q} \leftarrow \mathrm{A}_{s}^{*}\right),
$$

\footnotetext{
${ }^{4}$ For exciton-like branches, characterised by vanishing group velocity, the modes with $k \approx \pi / a$ might be in resonance with the frequency $\omega_{\mathrm{A}}{ }^{*}$ : These modes have not been accommodated in the presented theory concentrating on the modes with $k \ll \pi / a$ for which the description in terms of the dielectric constant is applicable.
} 
$\rho_{\mathrm{s}}$ being the population distribution of the initial state of the emitting molecule A. Now the transition frequencies $\omega_{\mathrm{A}^{*}}^{s q}$ and the transition dipole moments $\mu_{\mathrm{A}^{*}}^{q s}$ are to be supplied by the indexes $s$ and $q$.

Eq. (4.6) represents the refractive modification of the spontaneous emission rate in a dielectric medium. For dilute media $(n \rightarrow 1)$, the result (4.6) reduces to that familiar from the QED textbooks $[1,47,48]$. Eq. (4.6) is also consistent with the conclusions of the previous microscopic QED theories of spontaneous emission in the condensed phases $[3,4]$. Yet, the present study provides a more realistic representation of a dielectric medium than that of Refs. [3,4] in which the use has been made of one-frequency (two-level) representation for each molecule constituting the surrounding dielectric medium. In contrast, here an arbitrary number of molecular levels contributes to the refractive index $n$, defined through Eqs. (3.21) and (3.22). Note also, that the microscopically derived expression (4.6) supports the macroscopic approach in which the local field factors are phenomenologically introduced on the basis of virtual cavity arguments [49]. On the other hand, employment of a real cavity surrounding the embedded molecule, leads to a completely different result for the rate of spontaneous emission [50].

It is to be pointed out, although the local field corrections and other refractive contributions enter the transfer rate (4.6), the result does not reflect at this stage the modification of the molecular frequency $\omega_{\mathrm{A}^{*}}$ due to the medium. (The same applies to the previous microscopic considerations of the spontaneous emission by Knoester and Mukamel [3] and by Ho and Kumar [4].) Recent study by Juzeliunas and Andrews [36] showed that the molecular frequencies, featured in the energy-conservation delta-functions (as in Eqs. (4.3) and (4.4)) of the Fermi Golden Rule, are to be renormalised to represent the medium-induced level shifts for both the excited and ground states. In this way, the modification of the transition frequency $\omega_{\mathrm{A}^{*}}$ may be considered to be implicitly accommodated in the above formulas. The same applies to other molecule-radiation processes, including the linear absorption to be analysed next.

\subsubsection{Linear absorption}

Consider now the linear (one-"dressed photon") absorption, in which an imbedded molecule A is promoted to its excited state $\left|A^{*}\right\rangle$, as illustrated in Fig. 3. The initial and final states of such a process are constructed as

$$
\begin{aligned}
& |I\rangle=|\mathrm{A}\rangle \prod_{k, m, \lambda} \frac{\left(P_{k, m, \lambda}^{+}\right)^{q_{k, m, \lambda}}}{\left(q_{k, m, \lambda} !\right)^{1 / 2}}|0\rangle, \\
& \left|F_{k_{1}, m_{1}, \lambda_{1}}\right\rangle=\left|\mathrm{A}^{*}\right\rangle \frac{P_{k_{1}, m_{1}, \lambda_{1}}}{\left(q_{k_{1}, m_{1}, \lambda_{1}}\right)^{1 / 2}} \prod_{k, m, \lambda} \frac{\left(P_{k, m, \lambda}^{+}\right)^{q_{k, m, \lambda}}}{\left(q_{k, m, \lambda} !\right)^{1 / 2}}|0\rangle,
\end{aligned}
$$

where $q_{k, m, \lambda}$ is the number of medium-dressed photons (polaritons) in the mode $(k, m, \lambda)$ of the incident beam. The frequencies of the incident photons are again supposed to be distributed exclusively within the photonic branches of the dispersion, i.e., in the transparent areas of the molecular medium.

By making use of the Fermi Golden Rule, the rate of the process reads, through Eqs. (4.1) and (3.30)

$$
\begin{aligned}
& W\left(\mathrm{~A}^{*} \leftarrow \mathrm{A}\right) \\
& \quad=\sum_{k, m} \sum_{\lambda=1}^{2}\left(\frac{\pi \omega_{k}^{(m)} q_{k, m, \lambda} v_{\mathrm{g}}\left(\omega_{k}^{(m)}\right)}{\varepsilon_{0} c V_{0} n\left(\omega_{k}^{(m)}\right)}\right)\left(\frac{\left[n\left(\omega_{k}^{(m)}\right)\right]^{2}+2}{3}\right)^{2}\left[\boldsymbol{\mu}_{\mathrm{A}^{*}} \cdot \boldsymbol{e}^{(\lambda)}(\boldsymbol{k})\right]^{2} \delta\left(\hbar \omega_{\mathrm{A}^{*}}-\hbar \omega_{k}^{(m)}\right) .
\end{aligned}
$$

Performing also summation over the final sub-levels $s$ of molecule $\mathrm{A}$, and averaging over the initial ones denoted by $q$, the total rate of absorption takes the form

$$
\begin{aligned}
W^{\text {abs }} & =\sum_{s, q} \rho_{q} W\left(\mathrm{~A}_{s}^{*} \leftarrow \mathrm{A}_{q}\right) \\
& =\sum_{k, m} \sum_{\lambda=1}^{2} q_{k, m, \lambda} v_{\mathrm{g}}\left(\omega_{k}^{(m)}\right) \sigma_{\mathrm{A}}^{(k, m, \lambda)} / V_{0},
\end{aligned}
$$




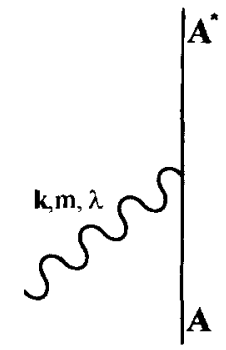

Fig. 3. Linear absorption.

where

$$
\sigma_{\mathrm{A}}^{(k, m, \lambda)}=\frac{1}{n\left(\omega_{k}^{(m)}\right)}\left(\frac{\left[n\left(\omega_{k}^{(m)}\right)\right]^{2}+2}{3}\right)^{2}\left(\frac{\pi \omega_{k}^{(m)}}{\varepsilon_{0} c}\right) \sum_{s, q} \rho_{q}\left[\boldsymbol{\mu}_{\mathrm{A}^{*}}^{s q} \cdot \boldsymbol{e}^{(\lambda)}(\boldsymbol{k})\right]^{2} \delta\left(\hbar \omega_{\mathrm{A}^{*}}^{s q}-\hbar \omega_{k}^{(m)}\right),
$$

$\rho_{q}$ being the thermal distribution function of the initial sub-levels of molecule A. Here the molecular sub-levels $q$ and $s$ have again been displayed explicitly in both the excitation frequencies $\omega_{\mathrm{A}^{t}}^{s q}=\left(\varepsilon_{\mathrm{A}^{*}}^{s}-\varepsilon_{\mathrm{A}}^{q}\right) / \hbar$ and the transition dipoles $\boldsymbol{\mu}_{\mathrm{A}}^{s q}$.

The group velocity $v_{\mathrm{g}}\left(\omega_{k}^{(\mathrm{m})}\right)$ enters the absorption rate (4.12), so that the quantity $q_{k, m, \lambda} v_{\mathrm{g}}\left(\omega_{k}^{(m)}\right) / V_{0}$ on the left represents a number of "medium-dressed" photons passing through the unit surface (normal to $k$ ) per unit time. In this way, $\sigma_{\mathrm{A}}^{(k, m, \lambda)}$ may be identified to be the absorption cross-section of A. It is obvious that $\sigma_{\mathrm{A}}^{(k, m, \lambda)}$ experiences the $n^{-1}\left[\left(n^{2}+2\right) / 3\right]^{2}$ modification in the medium. This is in agreement with results familiar from the classical electrodynamics of the condensed phases [49]. To our knowledge, it is the first time that the medium-induced modification of the absorption cross-section has been established on the basis of the microscopic QED approach.

The above analysis of the spontaneous emission and linear absorption may be extended to other absorption, emission or scattering processes (including the non-linear optical processes) by a guest molecular surrounded by a dielectric medium. This will be carried out in more details elsewhere.

\subsection{Two-centre (bimolecular) processes}

Consider next briefly bimolecular processes that involve two molecules A and B. The interaction Hamiltonian is now represented by two single-centre contributions, as

$$
V=-\varepsilon_{0}^{-1} \mu(\mathrm{A}) \cdot d^{\perp}\left(r_{\mathrm{A}}\right)-\varepsilon_{0}^{-1} \boldsymbol{\mu}(\mathrm{B}) \cdot d^{\perp}\left(r_{\mathrm{B}}\right) .
$$

Examples of such two-centre phenomena are resonance energy transfer [23-36], optical activity by a pair of achiral molecules [7,8], bimolecular single- or multi-photon absorption $[13,14,16]$ and others. In vacuum, bimolecular processes are described in terms of the intermolecular propagation of virtual photons. Mathematically, the bimolecular coupling is then represented through the familiar electromagnetic for the retarded dipole-dipole coupling tensor in vacuum (see, e.g. Refs. [28,31]). The present formalism describes the bimolecular coupling in another way through the intermolecular propagation of virtual polaritons. The retarded coupling tensor is then given by

$$
\begin{aligned}
\theta_{l p}(\omega)= & \frac{1}{\hbar \varepsilon_{0}^{2}} \sum_{k, m, \lambda}\left(\frac{\left\langle 0\left|d_{l}^{\perp}\left(r_{\mathrm{B}}\right)\right| \boldsymbol{k}, m, \lambda\right\rangle\left\langle k, m, \lambda\left|d_{p}^{\perp}\left(r_{\mathrm{A}}\right)\right| 0\right\rangle}{\omega-\omega_{k}^{(m)}+\mathrm{i} \eta}\right. \\
& \left.-\frac{\left\langle 0\left|d_{p}^{\perp}\left(r_{\mathrm{A}}\right)\right| \boldsymbol{k}, m, \lambda\right\rangle\left\langle\boldsymbol{k}, m, \lambda\left|d_{l}^{\perp}\left(\boldsymbol{r}_{\mathrm{B}}\right)\right| 0\right\rangle}{\omega+\omega_{k}^{(m)}-\mathrm{i} \eta}\right), \quad(\eta \rightarrow+0),
\end{aligned}
$$




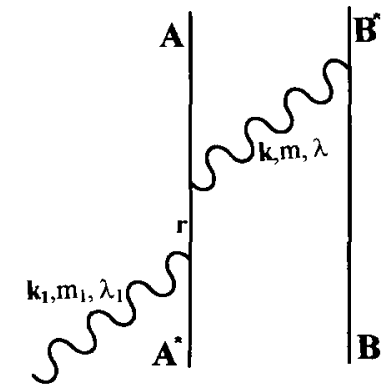

Fig. 4. A typical diagram for the laser-assisted energy transfer. A "dressed" laser photon is labelled by a set of mode indices ( $\left.k_{1}, m_{1}, \lambda_{1}\right)$, a virtual polariton being denoted as $(\boldsymbol{k}, \boldsymbol{m}, \lambda)$. Index $r$ labels intermediate states of $\mathrm{A}$.

where both the ground- (vacuum-) state vector $|0\rangle$, and the excited-state vectors $|\boldsymbol{k}, \boldsymbol{m}, \lambda\rangle$ are now dressed by a medium. In the previous study on resonance energy transfer [35], the above tensor was calculated in terms of the Green's function formalism, by-passing the explicit analysis of the operator for local displacement field. Alternatively, substituting the explicit mode expansion (3.30) for the local displacement operator into the tensor (4.14), one arrives now to the same modifications of $\theta_{\mathrm{lp}}(\omega)$ by the medium, as those presented in Eqs. (4.32)-(4.35) of the previous work [35].

The knowledge of the mode expansion makes it possible to deal also with the real incoming and outgoing photons, for which the explicit mode consideration cannot be by-passed. The real photons feature in higher-order bimolecular processes, such as in laser-assisted energy transfer [51] (depicted in Fig. 4), bimolecular absorption $[13,14,16]$, etc. Note, that each real photon brings the refractive modifications (given by Eq. (3.30)) that are to be calculated at the appropriate frequencies of absorbed or emitted photons. On the other hand, the frequency-dependent refractive index, entering the electromagnetic coupling tensor, is to be calculated at the frequencies of the appropriate mismatch energies. A more detail analysis of such higher-order bimolecular processes is presented in a separate study by Juzeliūnas and Andrews [52]. Finally, the mode expansion may be also be used for the description of the quantum dynamics, involving two and more centres, beyond the rate regime.

\section{Conclusion}

A microscopic QED theory has been developed for the description of molecule-radiation and moleculemolecule processes in condensed molecular media. Adopting a polariton model, an explicit normal-mode expansion has been derived for the operator of the local displacement field that describes the coupling of the individual molecules with the field. The expansion manifestly embodies refractive influences, including, inter alia, local field contributions. To this end, the local field effects intrinsically emerge within the present formalism that systematically treats photon Umklapp processes; in addition the theory accommodates an arbitrary number of molecular levels contributing to the refractive index. The mode expansion is applicable to a variety of molecule-radiation processes. Applications to the spontaneous emission and linear absorption, presented in the previous section, illustrate the approach. Applications to the two-centre processes have also been discussed. A more detail analysis of other molecule-radiation and molecule-molecule processes, including non-linear ones, will appear elsewhere. 


\section{References}

[1] D.P. Craig and T. Thirunamachandran, Molecular quantum electrodynamics (Academic Press, London, 1984).

[2] D.P. Craig and T. Thirunamachandran, Accounts Chem. Res. 19 (1986) 10.

[3] J. Knoester and S. Mukamel, Phys. Rev. A 40 (1989) 7065.

[4] S.-T. Ho and P. Kumar, J. Opt. Soc. Am. B 10 (1993) 1620.

[5] R.H. Dicke, Phys. Rev. 93 (1954) 99.

[6] D.P. Craig, E.A. Power and T. Thirunamachandran, Proc. Roy. Soc. A 348 (1976) 19.

[7] D.L. Andrews and and T. Thirunamachandran, Proc. Roy. Soc. A 358 (1977) 297.

[8] D.L. Andrews and and T. Thirunamachandran, Proc. Roy. Soc. A 358 (1977) 311.

[9] D.P. Craig and T. Thirunamachandran, Proc. Roy. Soc. London A 410 (1987) 337.

[10] D.P. Craig and T. Thirunamachandran, Intern. J. Quantum Chem. 31 (1987) 417.

[11] D.L. Andrews and W.J. Meath, J. Phys. B 26 (1993) 4633.

[12] D.L. Andrews, P. Allcock and A.A. Demidov, Chem Phys. Letters 231 (1994) 206.

[13] E. Hudis, Y. Ben-Aryeh and U.P. Oppenheim, Phys. Rev. A 43 (1991) 3631.

[14] D.L. Andrews and K.P. Hopkins, Advan. Chem. Phys. 77 (1990) 39.

[15] D.L. Andrews and N.P. Blake, Phys. Rev. A 41 (1990) 2547.

[16] G. Kweon and N.M. Lawandy, Phys. Rev. B 49 (1994) 4445.

[17] M.J. Stephen, J. Chem. Phys. 40 (1964) 669.

[18] R.R. McLone and E.A. Power, Matematika 11 (1964) 91.

[19] R.R. McLone and E.A. Power Proc. Roy. Soc. A 286 (1965) 573.

[20] D.P. Craig and P.D. Dacre, Proc. Roy. Soc. A 310 (1969) 297.

[21] D.P. Craig and L.A. Dissado, Proc. Roy. Soc. A 310 (1969) 313.

[22] E.A. Power and T. Thirunamachandran, Chem. Phys. 171 (1993) 1.

[23] D.A. Hutchinson and H.F. Hameka, J. Chem. Phys. 41 (1964) 2006.

[24] J.S. Avery, Proc. Phys. Soc. 88 (1966) 1.

[25] L. Gomberoff and E.A. Power, Proc. Phys. Soc. 88 (1966) 281.

[26] A.A. Serikov and Yu. M. Khomenko, Physica A 93 (1978) 383.

[27] E.A. Power and T. Thirunamachandran, Phys. Rev. A 28 (1983) 2671.

[28] D.L. Andrews and B.S. Sherborne, J. Chem. Phys. 86 (1987) 4011.

[29] D.L. Andrews, D.P. Craig and T. Thirunamachandran, Intern. Rev. Phys. Chem. 8 (1989) 37.

[30] D.P. Craig and T. Thirunamachandran, Chem. Phys. 135 (1989) 37.

[31] D.L. Andrews and G. Juzeliūnas, J. Chem. Phys. 95 (1991) 5513.

[32] D.P. Craig and T. Thirunamachandran, Chem. Phys. 167 (1992) 229

[33] G.D. Scholes, A.H.A. Clayton and K.P. Ghiggino, J. Chem. Phys. 97 (1992) 7405.

[34] G.D. Scholes and K.P. Ghiggino, J. Phys. Chem. 98 (1994) 4580.

[35] G. Juzeliūnas and D.L. Andrews, Phys. Rev. B 49 (1994) 8751.

[36] G. Juzeliūnas and D.L. Andrews, Phys. Rev. B 50 (1994) 13371.

[37] M. Orrit and P. Kottis, Advan. Chem. Phys. 74 (1988) 1.

[38] J.J. Hopfield, Phys. Rev. 112 (1958) 1555.

[39] V.M. Agranovich, Zh. Eksp. Teor. Fiz. 37 (1959) 430 [Soviet Phys. JETP 37 (1960) 307].

[40] A.S. Davydov and V.A. Onishchuk, Phys. Stat. Solidi 24 (1967) 373.

[41] R.K. Bullough and B.V. Thompson, J. Phys. C 3 (1970) 1780.

[42] M.R. Philpott, J. Chem. Phys. 52 (1970) 5842.

[43] K.T. Stoychev and M.T. Primatarowa, Phys. Rev. B 46 (1992) 10727.

[44] B. Huttner, J.J. Baumberg and S.M. Barnett, Europhys. Letters 16 (1991) 177.

[45] B. Huttner and S.M. Barnett, Europhys. Letters 18 (1992) 487.

[46] B. Huttner and S.M. Barnett, Phys. Rev. A 46 (1992) 4306.

[47] E.A. Power, Introductory quantum electrodynamics (Longmans, London, 1964).

[48] W.P. Healy, Non-relativistic quantum electrodynamics (Academic Press, London, 1982).

[49] V.M. Agranovich and M.D. Galanin, Electronic excitation energy transfer in condensed matter (North-Holland, Amsterdam, 1982).

[50] R.J. Glauber and M. Lewenstein, Phys. Rev. A 43 (1991) 467.

[51] P.L. Knight, J. Phys. B 10 (1977) L195.

[52] G. Juzeliūnas and D.L. Andrews, Chem. Phys., to be published. 